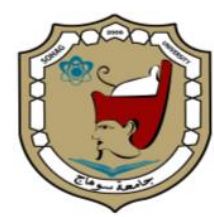

Sohag University

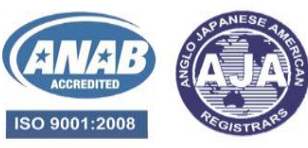

Sohag Medical Journal

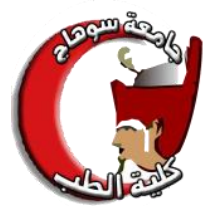

Faculty of Medicine

\title{
Causes of Pain in Knee Osteoarthritis
}

\section{Mohamed Hassan Mohamed Khalifa, Ahmed Roshdy Al-Agamy Radwan and Abdullah Mohammed Ahmed Radwan}

\author{
Department of Rheumatology \& Rehabilitation, Sohag Faculty of Medicine, Sohag \\ University
}

\begin{abstract}
Introduction: Osteoarthritis $(\mathrm{OA})$ is a common joint disorder, with the knee being one of the most frequently involved sites. Knee OA causes pain and stiffness and can lead to considerable disability and consequently to impaired quality of life.

Aim of the work: Clinical evaluation of participant with painful knee OA to detect the possible causes of pain knee OA.

Patients and Methods: Our study included 50 knee OA patients, attending to Sohag university hospitals rheumatology and rehabilitation outpatient clinic. They were divided according to pain into 2 groups. Group 1 with painful OA and group 2 with painless OA. Both groups were age and sex-matched.

Results: Our study showed that the group 1 participants had more varus deformities, more and significant effusion and Baker cyst. Among painful OA patients, all factors showed non-significant relation to the degree of VAS.

Conclusion: Our study found that knee effusion and Baker cyst were the main possible causes of pain in knee OA.
\end{abstract}

Keywords: Knee, Osteoarthritis, Pain

\section{Introduction}

Osteoarthritis (OA) is the commonest joint inflammation. The knee is one of the most commonly involved joints; especially in Egypt. Pain is a common symptom that can lead to substantial disability and impaired quality of life. The pain source in knee osteoarthritis (OAK) is not properly demarcated. Moreover, the degree of $\mathrm{OA}$ is not corresponding to the degree of pain. These findings assume that there are other possible causes for pain different from cartilage pathology. The determination of the source of pain in OAK is so very important (1).

It is highly presumed that pain in OAK is multifactorial, with bone, cartilage and soft tissue changes are accused.
Obesity and joint mal-alignment may also be involved. Low degree of synovitis may also have a role. Altered sensitivity of the peripheral or central nerves may play a role (2).

In this study, we tried to detect the cause of pain in OAK, using only clinical assessment of OAK patients.

\section{Patients and Methods:}

\section{Ethical considerations:}

The study was approved by the Scientific Ethical Committee of Faculty of Medicine, Sohag University. Informed written consent was acquired from all of the study participants.

Patients: Fifty participants attending to Sohag University Hospitals Rheum- 
SOHAG MEDICAL JOURNAL

Vol. 24 No. 3 July 2020

atology and Rehabilitation outpatient clinic with clinically determined OAK. Depending on the pain they were divided into two groups (each of which contain 25 patients):

- Group A: patients with knee pain during physical activity once at least in the previous 3 days prior to inclusion.

- Group B: patients without knee pain from at least 1 month prior to inclusion.

Participants with severe knee OA, secondary OA, 6 months history of trauma, knee replacement surgery, other chronic rheumatic disease and intraarticular injection 3 months before the study were excluded from the study.

Methods: All of the participants were subjected to full history (demographic data and personal history, detailed history of general health condition and chronic or current diseases), general examination, knee clinical examination and knee alignment were assessed.

Pain was measured using the Visual analogue scale (VAS) on a 0-100 scale, with zero means "no pain" and 100 means "worst possible pain" (3-5). Each patient was asked to give a number for his pain or to point at a graded ruler.

\section{Statistical analysis:}

- Statistical package for social sciences (IBM-SPSS), version 25 (IBMChicago, USA; 2017) was used for statistical data analysis.

- Data expressed as mean, standard deviation (SD), number and percentage. Mean and standard deviation
Causes of Pain in Knee Osteoarthritis

Ahmed Roshdy Al-Agamy

were used as a descriptive value for quantitative data, while number and percentage were used to describe qualitative data.

- Student t-test was used to compare the means between two groups, and Pearson Chi-square was used to compare percentages of qualitative data. Pearson correlation test was used to compare two quantitative variables. The value of (r) is explainned as: $\mathrm{r}<0.4 \rightarrow$ weak correlation, $0.4-0.7 \rightarrow$ moderate correlation, $0.7-1 \rightarrow$ strong correlation. If the (r) is positive $\rightarrow$ direct correlation and if negative $\rightarrow$ inverse correlation.

- For all these tests, the level of significance (P-value) can be explained as being significant if $<0.05$.

\section{Results}

Our study showed that the mean age of the study groups was around 52-55 years, with a non-significant difference between the two groups. Females were slightly more predominant among the painful group (60\%) than among controls (52\%). Painful cases had higher BMI (24.6) than controls (23.8). The painful group had more but non significant varus deformities ( $28 \%$ compared to only $8 \%$; respecttively). In fact, we did not report any valgus deformity amog any of our cases in both groups. Also, the painful group showed more and significant effusion $(76 \%$ compared to $28 \%)$ and Baker cysts (16\% versus zero) (Table 1). 


\begin{tabular}{|c|c|c|c|c|}
\hline \multicolumn{2}{|l|}{ Item } & Active & Control & P-value \\
\hline Age & Mean \pm SD & $51.72 \pm 10.00$ & $55.32 \pm 12.01$ & 0.255 (NS) \\
\hline \multirow[t]{2}{*}{ Sex } & Male & $10(40 \%)$ & $12(48 \%)$ & \multirow[t]{2}{*}{0.569 (NS) } \\
\hline & Female & $15(60 \%)$ & $13(52 \%)$ & \\
\hline BMI & Mean \pm SD & $24.57 \pm 3.09$ & $23.78 \pm 3.17$ & $0.337(\mathrm{NS})$ \\
\hline \multirow[t]{2}{*}{ The most affected joint } & Right & $9(36 \%)$ & $14(56 \%)$ & $0.156(\mathrm{NS})$ \\
\hline & Left & $16(64 \%)$ & $11(44 \%)$ & \\
\hline VAS & Mean \pm SD & $46.40 \pm 4.21$ & $\mathbf{0}$ & $<0.001(\mathrm{HS})$ \\
\hline \multirow[t]{3}{*}{ Alignment } & Normal & $18(72 \%)$ & $23(92 \%)$ & $0.066(\mathrm{NS})$ \\
\hline & Varus deformity & $7(28 \%)$ & $2(8 \%)$ & \\
\hline & Valgus deformity & $\mathbf{0}$ & $\mathbf{0}$ & \\
\hline Effusion & & 19(76\%) & $7(28 \%)$ & $0.001(\mathbf{S})$ \\
\hline Baker's cyst & & $4(16 \%)$ & $\mathbf{0}$ & $0.037(\mathbf{S})$ \\
\hline
\end{tabular}

\section{Table 1. Case/control comparison regarding demographic data}

Relating the pain (measured by VAS) and other demographic and clinical data among the painful OAK group only revealed that non of these factors is associated with significantly increased degree of pain. In fact, advanced age and higher BMI were weakly correlated with more pain, females had a little more pain. Regading the laterality, no difference could be detected regarding the effect on pain. Varus deformity and Baker's cyst were associated with more pain, but still non significant (Table 2).

\begin{tabular}{|c|c|c|c|}
\hline \multicolumn{2}{|c|}{ Item } & \multirow{2}{*}{$\begin{array}{c}\text { Mean VAS } \\
0.252\end{array}$} & \multirow{2}{*}{$\begin{array}{c}\text { P-value } \\
0.224(\mathrm{NS})\end{array}$} \\
\hline Age & Correlation & & \\
\hline \multirow[t]{2}{*}{ Sex } & Male & $45.00 \pm 5.27$ & $0.180(\mathrm{NS})$ \\
\hline & Female & $47.33 \pm 3.20$ & \\
\hline BMI & Correlation & 0.126 & $0.549(\mathrm{NS})$ \\
\hline \multirow{2}{*}{ The most affected joint } & Right & $46.67 \pm 5.00$ & $0.818(\mathrm{NS})$ \\
\hline & Left & $46.25 \pm 3.87$ & \\
\hline \multirow[t]{2}{*}{ Alignment } & Normal & $43.94 \pm 9.74$ & \multirow[t]{2}{*}{$0.149(\mathrm{NS})$} \\
\hline & Varus & $51.43 \pm 14.73$ & \\
\hline \multirow[t]{2}{*}{ Effusion } & Yes & $45.79 \pm 4.49$ & \multirow[t]{2}{*}{$0.204(\mathrm{NS})$} \\
\hline & No & $48.33 \pm 2.58$ & \\
\hline \multirow[t]{2}{*}{ Baker cyst } & Yes & $48.75 \pm 2.50$ & \multirow[t]{2}{*}{$0.231(\mathrm{NS})$} \\
\hline & No & $45.95 \pm 4.36$ & \\
\hline
\end{tabular}

Table 2. The relation between pain (measured by VAS) and clinical and ultrasonographic data of the study cases 


\section{Discussion}

In this study, we compared the clinical findings between painful and non painful OAK patients to detect the cause of pain in OA.

Our study included 50 OAK patients. The mean age of the study groups in our study showed a non-significant difference between the two groups. Females were more predominant among cases but this was also non significant. Our findings agreed to the results of $\boldsymbol{E} \boldsymbol{l}$ Monaem et al. 2017 (6), whose patients ranged in age between 40 and 66 years with a mean age of $51.9 \pm 7.82$ years; but they showed more female predominance with 160 females $(80 \%)$ and 40 males $(20 \%)$. Our patients were, however, younger than those studied by Kiadaliri et al. 2016 (7) whose patients' mean age was around 71.5 years for non painful $\mathrm{OA}$ and 70.1 years for painful OA. Also, They found female predominance, with females accounted for $61 \%$ of non painful OA and $64 \%$ of painful OA.

We found a non significantly higher BMI in cases than controls. These results were agreed with the results Kiadaliri et al. 2016 (7) showed non significant difference between painful and painless OA cases, with BMI of 28.8 and 28.9; respectively. On the other hand, our results were different from that of El Monaem et al. 2017 (6) who showed statistically significant correlations between painful OAK and each of BMI, osteophyte and quadriceps tendon thickness. Also, our results were agreed with the study of Maly $\boldsymbol{e t}$ al. 2008 (8) who found a significant and positive relation between body weight and pain among OAK patients.

In our study, malalignment was more seen among cases compared to controls; with a near significant difference. The study of Maly et al. 2008 (8) on the opposite hand, failed to find any significant correlation between pain and malalignment. However, they used WOMAC score to measure pain in stead of VAS.

We found that effusion and Baker's cyst were the only two factors which were significantly more among cases compared to controls. This agreed with that study done by El Monaem et al. 2017 (6) who found significant correlations between Baker's cyst size and pain score, which is in accordance with the observation of Chiba et al. 2016 (9) who documented that painful flare-ups in knee OA frequently arise from Baker's cysts.

\section{Conclusion:}

In our study, we found that effusion and Baker cyst were the only two factors associated with painful OAK. This study should be extended to include imaging evaluation on higher number of cases.

\section{References:}

1. Bevers K, Bijlsma JW, Vriezekolk JE, van den Ende $\mathrm{CH}$, den Broeder AA. Ultrasonographic features in symptomatic osteoarthritis of the knee and relation with pain. Rheumatology (Oxford). 2014;53(9):1625-9.

2. O'Neill TW, Felson DT. Mechanisms of Osteoarthritis (OA) Pain. Current osteoporosis reports. 2018;16(5):611-6.

3. Elsaman AM, Radwan AR, Mohammed WI, Ohrndorf S. Low-dose spironolactone: treatment for osteoarthritis-related knee effusion. A prospective clinical and sonographicbased study. The Journal of rheumatology. 2016;43(6):1114-20.

4. Carlsson AM. Assessment of chronic pain. I. Aspects of the reliability and validity of the visual analogue scale. Pain. 1983;16(1):87-101. 
SOHAG MEDICAL JOURNAL

Vol. 24 No. 3 July 2020

5. Crichton N. Visual analogue scale (VAS). J Clin Nurs. 2001;10(5):706-6.

6. El Monaem SMA, Hashaad NI, Ibrahim NH. Correlations between ultrasonographic findings, clinical scores, and depression in patients with knee osteoarthritis. European journal of rheumatology. 2017;4(3):205.

7. Kiadaliri AA, Lamm CJ, de Verdier MG, Engström G, Turkiewicz A, Lohmander LS, et al. Association of knee pain and different definitions of knee osteoarthritis with health-related quality of life: a population-based cohort study in southern Sweden.
Causes of Pain in Knee Osteoarthritis Ahmed Roshdy Al-Agamy

Health and quality of life outcomes. 2016;14(1):121.

8. Maly MR, Costigan PA, Olney SJ. Mechanical factors relate to pain in knee osteoarthritis. Clinical biomechanics. 2008;23(6):796-805.

9. Chiba D, Tsuda E, Maeda S, Sasaki E, Takahashi I, Nakaji $S$, et al. Evaluation of a quantitative measurement of suprapatellar effusion by ultrasonography and its association with symptoms of radiographic knee osteoarthritis: a cross-sectional observational study. Arthritis research \& therapy. 2016;18(1):181. 